\title{
Rousseau et les philosophes. Sous la direction de Michael O'Dea
}

\section{Peter Balazs}

\section{Q OpenEdition}

10 Journals

\section{Édition électronique}

URL : https://journals.openedition.org/studifrancesi/4694

DOI : 10.4000/studifrancesi.4694

ISSN : 2421-5856

\section{Éditeur}

Rosenberg \& Sellier

\section{Édition imprimée}

Date de publication : 1 avril 2012

Pagination : 149-150

ISSN : 0039-2944

\section{Référence électronique}

Peter Balazs, «Rousseau et les philosophes. Sous la direction de Michael O'Dea », Studi Francesi [En

ligne], 166 (I | LVI) | 2012, mis en ligne le 30 novembre 2015, consulté le 18 novembre 2021. URL :

http://journals.openedition.org/studifrancesi/4694 ; DOI : https://doi.org/10.4000/studifrancesi.4694

Ce document a été généré automatiquement le 18 novembre 2021.

\section{(c) (i) (9)}

Studi Francesi è distribuita con Licenza Creative Commons Attribuzione - Non commerciale - Non opere derivate 4.0 Internazionale. 


\title{
Rousseau et les philosophes. Sous la direction de Michael O’Dea
}

\author{
Peter Balazs
}

\section{RÉFÉRENCE}

Rousseau et les philosophes. Sous la direction de Michael o’DEA. Oxford, Voltaire Foundation, 2010, pp. 274.

Cet ouvrage collectif, qui reprend les communications présentées au colloque de l'Association Rousseau, tenu à Lyon en juillet 2007, traite du rapport complexe et contradictoire qu'entretient Jean-Jacques avec ses amis/ennemis que sont les Philosophes, ainsi que de la place très controversée qu'il occupe dans la philosophie des Lumières. Dans son «Introduction», Michael O' Dea donne le ton du volume en présentant rapidement d'un aspect personnel le rapport de Rousseau avec la côterie diderotienne. Dans son article, Sur le concept de lumières publiques: Rousseau comme Aufklärer (pp.19-28), Bruno BERNARDI se demande, sur les traces de Mark Hulliung, si Rousseau appartient à la philosophie des Lumières, s'il est philosophe ou antiphilosophe, et enfin, s'il oblige ses anciens amis à s'aligner en partie sur ses positions ou bien s'il s'agit d'une influence quasi réciproque que Diderot et Rousseau s'exercent l'un sur l'autre. Pour Bernardi, si Rousseau s'inscrit clairement dans l'horizon des Lumières et si sa pensée doit être considérée comme une auto-critique du mouvement, il est indiscutable que pour lui l'émergence des lumières publiques requiert également des conditions passionnelles (et non seulement rationelles). Christophe BERTRAM (Rousseau and morality: between naturalism and rationalism, pp. 29-37), quant à lui, cherche à situer la pensée de Rousseau par rapport au naturalisme de Hume et au rationalisme kantien. Bertram conclut que les lois que les hommes-devenus citoyens-se donnent ne sont pas celles d'un être parfaitement rationnel, mais d'humains qui occupent une sorte de position intermédiaire entre l'être pré-moral du second Discours et une moralité idéale. James sWENSON (Du matérialisme chez Rousseau, 
pp. 38-47) étudie l'éventuel «matérialisme» du plus antimatérialiste des philosophes, qui avait néanmoins un ouvrage inachevé intitulé La morale sensitive ou le matérialisme du sage... Swenson - qui précise d'abord que par anti-matérialisme nous devons entendre anti-déterminisme-conclut à l'existence dans la pensée de Rousseau de mondes antimatérialistes, des hypothèses méthodologiques qui prouvent que dans le monde il existe des formes de servitude (la vie dans la cour ou en ville) qui éliminent la liberté et font de l'homme un véritable automate. Christophe VAN STAEN (Un océan de connaissances inutiles: Rousseau face aux Glaucus modernes, pp. 47-56) étudie l'histoire de la statue de Glaucus, image célèbre du second Discours, empruntée soit à Platon (République), soit à Ovide (Métamorphoses). La fonction de cette fable, allégorie de la connaissance de soi, est analysée ici dans le contexte d'une comparaison des finalités que Rousseau et les Encyclopédistes accordent à la philosophie: finalité proprement humaine pour le premier, projet baconien de la maîtrise de la nature pour Diderot et ses amis.

2 La distinction entre l'homme corrompu par la civilisation et celui qui est près de la nature est au centre de l'étude de Christopher KELLy (Rousseau and the bad calculations of Philosophers, pp. 57-66). Rousseau non seulement n'accepte pas l'appréciation désabusée de la vie par Maupertuis («La vie est un assés mauvais présent»), fondée sur le calcul des biens et des maux, mais il déconseille cette philosophie, jugée superficielle, à sa correspondante, une certaine Henriette. Alexandre PROVENCHER-GRAVEL (Une philosophie pour les autres?, pp.67-74) étudie la troisième promenade des Rêveries: il souligne la rapidité par laquelle Rousseau passe sur les questions de fond, pour faire de la sincérité et de l'utilité le critère principal de la vérité. L'auteur se demande néanmoins si le promeneur solitaire mène vraiment sa recherche ardue muni seulement de sa bonté naturelle et de tout ce qui en découle. Dans une étude très intéressante de point de vue méthodologique (L'art de ces Messieurs: Rousseau et la question herméneutique, pp. 77-98), Jean-François PERRIN oppose deux œuvres de Rousseau, celle que Jean-Jacques lui-même tente de construire et celle qu'ont élaborée ses ennemis. Or est-il possible, se demande Rousseau, de détourner les lecteurs postérieurs de ses ouvrages et de les convertir à l'interprétation voulue par lui? Catherine vOLPILHAC-AUGER, quant à elle, rappelle un passage auto-biographique de l'Émile (Tais-toi, Jean-Jacques. D"Émile" aux "Dialogues", pp. 99-108). Rousseau s'entend dire par une voix féminine: «Tais-toi Jean-Jacques, ils ne te comprendront pas». Injonction maternelle ou ordre venu d'en haut? De toute manière une phrase comportant un élément affectif et quasi religieux. Michael o'DEA (Soundings: the word "philosophe» in Rousseau's work and correspondence, pp. 109-130) étudie l'œuvre de Rousseau à travers son emploi du mot philosophe et du champ lexical qui l'entoure. Le fait que le terme ne devient jamais uniformément négatif illustre que la position de Rousseau par rapport aux causes de son malheur était pour le moins ambigue: tantôt il désigne Diderot et les philosophes comme les responsables principaux de sa solitude, tantôt il attribue à Choiseul le rôle du bouc émissaire. Caroline L. MINEAU (La fiction de l'autre dans le portrait de soi: le rôle des figures de Grimm, de Diderot et d'Holbach dans les "Confessions", pp.131-140) évalue la présentation par Rousseau de ses amis devenus ennemis acharnés. Le rôle qu'il attribue à Grimm est de loin le moins enviable: c'est ce dernier qui est présenté comme l'homme dont l'âme est sur tous les points opposée à la sienne.

3 L'article d'Ourida mostefaI (Une dette non-avouée: Rousseau, Diderot et l'Encyclopédie, pp.141-150) souligne que dans les Confessions, Rousseau s'efforce de minimiser et de déprécier sa collaboration à l'Encyclopédie, ouvrage dont il ne cesse d'ailleurs de se 
servir après sa rupture avec ses anciens amis. Même La lettre sur les spectacles, texte de rupture s'il en fut, porte l'empreinte des idées des Encyclopédistes. Kiyotaka KAWAI (Rousseau citoyen de Genève et sa critique du système représentatif, pp. 151-158) montre que dans le domaine de la théorie de la représentation Rousseau, à première vue, s'oppose le plus catégoriquement possible à la plupart de ses contemporains: en vérité, en plusieurs ouvrages (comme par exemple dans les Considérations sur le gouvernement de Pologne), il se verra contraint de modérer ses exigences de démocratie directe. L'étude de Carole MARTIN (Formes de l'empathie chez Rousseau et Diderot: réciprocité ou compassion, pp.161-174) montre en quoi l'esthétique visuelle de Rousseau diffère de celle de Diderot: chez le premier c'est dans la reconnaissance de sa conformité avec autrui qu'advient la pleine conscience de soi, comme le montre la célèbre scène des Confessions avec Mme Basile, où l'encadrement s'abolit vite pour laisser la place à la parfaite réciprocité des sentiments. John T. sсотт (Another dangerous supplement: Diderot's dialogue with Rousseau in the SVB, pp.175-193.), quant à lui, reprend la question du "rousseauisme" du Supplément au voyage de Bougainville de Diderot, un texte qui met en relief la difficulté insurmontable d'isoler tout ce qui est nature de ce qui ne l'est pas. On découvre, grâce à cet article bien documenté, que les Tahitiens, dans un sens plus libres que les Européens, sont également socialisés et soumis à un certain nombre d'obligations.

4 L'étude de Maria LEONE ( La Nouvelle-Héloïse et ses lecteurs philosophes: quand l'écriture romanesque redéfinit les modalités du dialogue de Rousseau et des ses "ennemis", pp. 193-204) analyse le dispositif paratextuel de la Nouvelle Héloise afin d'explorer les potentialités philosophiques de l'œuvre. S'agirait-il d'une quasi-réponse à la célèbre lettre de Voltaire dans laquelle il affiche son refus de prendre pour sérieux le second Discours de Rousseau? Le texte de Julia SIMON (Rousseau and the philosophes on commerce, pp. 205-214) met en relief à quel point Rousseau s'écarte du consensus que représente l'article SOCIÉTÉ de l'Encyclopédie, tiré de Burlamaqui. L'auteur du Contrat social ne fait pas grande confiance à la «communication réciproque que les hommes se font» et propose aux Corses et aux Polonais de limiter au strict nécessaire leurs rapports au-delà de la famille et de se méfier de l'argent. Nathan MARTIN (Rousseau and the philosophes on music history, pp. 215-224) confronte les ouvrages musicologiques de Rousseau à ceux de ses contemporains et il montre que l'auteur de la Lettre sur la musique française a, contrairement à ses confrères dans le domaine, consulté un large corpus de manuscrits anciens, afin de prouver que Rameau avait tort: l'harmonie n'est pas la base universelle et nécessaire de la musique, mais plutôt un phénomène contingent et historique. Martin STERN ("Voilà la véritable philosophie»: les conversions de Rousseau comme clef d'intelligibilité de sa relation aux philosophes, pp. 225-232) étudie les conversions de Rousseau, dont la plus importante est sans doute l'illumination de Vincennes, afin de souligner qu'on ne doit pas voir chez lui de véritable metanoia: il est plutôt empêtré dans des contradictions, ce qui explique en partie le caractère tendu des rapports qu'il entretient avec ses anciens amis. Le volume se clôt avec l'étude de Mark HULLIUNG (Rousseau and the philosophes: facing up to the Enlightenment wars, pp.235-251), qui propose un regard rétrospectif sur les critiques dont les Lumières ont été l'objet depuis la seconde guerre mondiale. Dans cette étude, qui reprend les idées de Talmon, AdornoHorkheimer, Gay, Darnton, Judt et Wokler, Rousseau n'est jamais entièrement séparé des philosophes ses contemporains: son œuvre est considérée comme l'autocritique des Lumières et non pas comme une critique venue d'extérieur. 